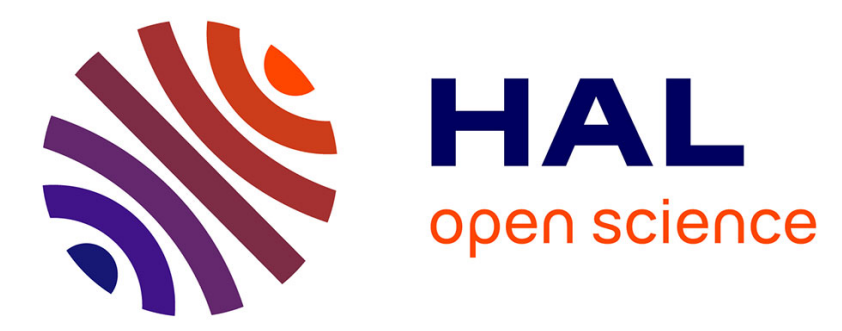

\title{
Correlation between structural properties and resistivity critical behavior in SrRuO3 thin films
}

Alice Galdi, P. Orgiani, L. Maritato, Laurence Méchin

\section{To cite this version:}

Alice Galdi, P. Orgiani, L. Maritato, Laurence Méchin. Correlation between structural properties and resistivity critical behavior in SrRuO3 thin films. Journal of Physics: Condensed Matter, 2012, pp.435603. hal-00976091

\section{HAL Id: hal-00976091 \\ https://hal.science/hal-00976091}

Submitted on 9 Apr 2014

HAL is a multi-disciplinary open access archive for the deposit and dissemination of scientific research documents, whether they are published or not. The documents may come from teaching and research institutions in France or abroad, or from public or private research centers.
L'archive ouverte pluridisciplinaire HAL, est destinée au dépôt et à la diffusion de documents scientifiques de niveau recherche, publiés ou non, émanant des établissements d'enseignement et de recherche français ou étrangers, des laboratoires publics ou privés. 


\title{
Correlation between structural properties and resistivity critical behavior in $\mathrm{SrRuO}_{3}$ thin films
}

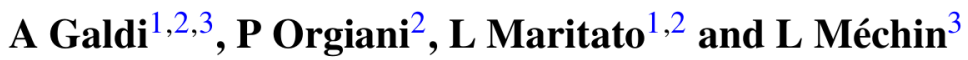 \\ ${ }^{1}$ DIIN Department of Industrial Engineering, Università degli Studi di Salerno, Via Ponte don Melillo, \\ I-84084 Fisciano (SA), Italy \\ ${ }^{2}$ CNR-SPIN, UOS Salerno, Via Ponte don Melillo, I-84084 Fisciano (SA), Italy \\ ${ }^{3}$ GREYC, ENSICAEN, UCBN, CNRS, UMR 6072, 6 bd du Maréchal Juin, F-14050 Caen Cedex, \\ France
}

E-mail: agaldi@unisa.it

\begin{abstract}
$\mathrm{SrRuO}_{3}$ is a strong itinerant ferromagnet showing many features of 'bad metals' together with Fermi liquid behavior at very low temperature. The intriguing magnetic and transport properties of $\mathrm{SrRuO}_{3}$ are tightly related to structure, as commonly observed in transition metal oxides. Here we report on the correlation of structural parameters (lattice constant and surface roughness) with the critical behavior of resistivity at the Curie point in $\mathrm{SrRuO}_{3}$ thin films deposited on (001) $\mathrm{SrTiO}_{3}$ by PLD. By varying the deposition conditions we were able to obtain a wide variety of structural properties in our samples, thus allowing us to perform a systematic study. Our analysis demonstrates the direct correlation between the critical temperature $T_{\mathrm{P}}$ and the lattice out-of-plane parameter. Furthermore, the value of the critical exponent $\lambda$ is proved to be a good physical parameter to quantify the microscopic order of $\mathrm{SrRuO}_{3}$ samples.
\end{abstract}

\section{Introduction}

The $\mathrm{SrRuO}_{3}$ (SRO) compound is a distorted perovskite ferromagnetic oxide (with a Curie temperature $T_{\mathrm{C}} \approx 165 \mathrm{~K}$ in bulk) characterized by a metallic behavior in the whole explored temperature range. Because of its low resistivity among perovskite structure oxides (the single crystal resistivity is about $150-300 \mu \Omega \mathrm{cm}$ at room temperature), it is one of the best conducting metallic oxides. Furthermore its chemical stability (up to $1200 \mathrm{~K}$ ) and the compatibility of its structure with many functional oxides (i.e. $\mathrm{YBa}_{2} \mathrm{Cu}_{3} \mathrm{O}_{6-x}$, $\mathrm{BaTiO}_{3}$ ), make SRO a good candidate to be used as metallic base electrode or buffer layer in all-oxide electronic devices. Consequently a number of studies have been reported on the growth mechanism and optimization of SRO thin films $[1,2]$.

SRO is a $4 d$ transition metal oxide, whose magnetism, of itinerant character, is determined by the antibonding $\pi \mathrm{pd}$ conduction bands originating by the hybridization of $\mathrm{Ru}$ and $\mathrm{O}$ orbitals. The exchange splitting and the magnetic ground state itself critically depend on the $\mathrm{Ru}-\mathrm{O}-\mathrm{Ru}$ bond angle (i.e. on the $\mathrm{RuO}_{6}$ octahedron rotations in the distorted perovskite structure), that affect the bandwidth of the conduction band and lead to pseudogap opening [3]. This is demonstrated by the non-magnetic compounds $\mathrm{CaRuO}_{3}$ and $\mathrm{BaRuO}_{3}$ (where isovalent cations substitute $\mathrm{Sr}$ ), whose structure slightly differs from the SRO one just because of slight tiltings and rotations of $\mathrm{RuO}_{6}$ octahedra. SRO thin film magnetic properties are therefore very sensitive to epitaxial strain and microstructure [4], and due to the strong coupling between electrons, phonons and magnons [3], this also affects the transport properties [5].

Several authors pointed out the 'bad metal' nature of SRO, revealed by the non-saturated resistivity even at temperatures of $1000 \mathrm{~K}$ [6] (well beyond the Ioffe-Regel limit) [7], the enhanced low temperature specific heat [8] and the coexistence of itinerant band magnetism with a sharp 
kink in the resistivity at $T_{\mathrm{C}}$ [9]. In elemental ferromagnets the magnetic part of the scattering originates a kink in the $\rho(T)$ curve at $T_{\mathrm{C}}$. According to the work of Fisher and Langer [10], this anomaly consists in a divergence in the $\mathrm{d} \rho(T) / \mathrm{d} T$ characterized by a critical exponent $\lambda$ that coincides with $\alpha$, the critical exponent of the specific heat $C_{p}$ (for $T>T_{\mathrm{C}}, \alpha \approx 0.1$ ), as verified experimentally on $\mathrm{Ni}$ and $\mathrm{Gd}$ (i.e. the anomalous part of resistivity is proportional to the magnetic internal energy). In contrast, the $\mathrm{d} \rho(T) / \mathrm{d} T$ divergence observed on SRO thin films and single crystals is much stronger at $T>T_{\mathrm{C}}$ with critical exponent $\lambda=0.5-0.9$, and weaker (logarithmic) at $T<T_{\mathrm{C}}$, in apparent contradiction with the Fisher and Langer result [8,9]. This behavior, contrasting with the apparently canonical critical exponents of the magnetization [9], has been attributed to the non-Fermi liquid behavior of this compound. In the present work we discuss the correlation between the structure/morphology and the transport properties of a large series of SRO thin film samples deposited by pulsed laser deposition (PLD), with a particular focus on the critical behavior of $\rho(T)$.

\section{Experiment}

SRO thin films have been deposited by pulsed laser deposition (PLD) on $\mathrm{SrTiO}_{3}$ (STO)(001) substrates. The crystal structure of SRO is orthorhombic with Pbnm symmetry and lattice parameters $a=0.55670, b=0.55304, c=0.78446 \mathrm{~nm}$, corresponding to a pseudocubic unit cell with lattice parameter $a_{\mathrm{pc}} \approx 0.393 \mathrm{~nm}$, so that $\mathrm{SRO}$ is subject to compressive strain on STO $\left(a_{\mathrm{pc}}=0.3905 \mathrm{~nm}\right)$. The SRO preferentially grows (110) oriented on (001) STO. The SRO [110] and [001] directions align either with STO[010] or [100], giving rise to in-plane twinning that reduces the crystalline quality of thin films. To suppress twinning, vicinal substrates with steps along $\mathrm{STO}\langle 100\rangle$ directions proved to be effective, allowing the production of single domain films with narrow rocking curves if compared to the ones grown on exact substrates [11]. However the use of vicinal substrates can induce transport anisotropy in thin samples [12, 13], especially in non-optimized conditions, when the growth mode is not purely 2D. For the sake of our study, the use of exact substrates allows us to both introduce different sources of disorder in the system and to limit the occurrence of transport anisotropies.

The films were grown in oxygen pressures ranging from 0.1 and 0.5 mbar, at temperatures ranging from 700 and $740{ }^{\circ} \mathrm{C}$. A $\mathrm{KrF}$ excimer laser was used, producing pulses of $30 \mathrm{~ns}$; the pulses are focused on the target to a spot of $\sim 3 \mathrm{~mm}^{2}$, and the target-substrate distance was kept fixed at $50 \mathrm{~mm}$. The energy and frequency of the pulses were varied in order to tune the deposition rate ( $E=150-200 \mathrm{~mJ}, f=1-3 \mathrm{~Hz}$ ). After the deposition, in order to oxygenate the film, the deposition chamber was filled with oxygen at 700 mbar and the sample was cooled down to room temperature at a rate of $-10^{\circ} \mathrm{C} \mathrm{min}^{-1}$. All samples were measured by $\mathrm{x}$-ray diffraction (XRD) in the Bragg-Brentano configuration in order to measure the out-of-plane lattice parameter and estimate the film thickness by finite size oscillations. Reciprocal space maps (RSM) of selected samples were collected around the (103) STO reflection in order to measure the in-plane lattice parameter of SRO. The RSM were performed by a four-circle Philips $X$ 'Pert diffractometer using $\mathrm{Cu} \mathrm{K}_{\alpha}$ radiation and equipped with a double $\mathrm{Ge}(220)$ monochromator.

The surface roughness of the films was determined by tapping mode atomic force microscopy (AFM). The root mean squared roughness value $R_{q}$, defined as the standard deviation of the height on a given area, was measured on 1,2 and $5 \mu \mathrm{m}$ side areas, and the resulting values averaged.

Resistance versus temperature $(R(T))$ curves were collected in a closed cycle cryocooler from 10 to $300 \mathrm{~K}$, controlled by means of a resistive heater in order to have a ramp rate of $3 \mathrm{~K} \mathrm{~min}^{-1}$. The $R(T)$ was measured in DC bias pulse reversed mode with pulse length of $330 \mathrm{~ms}$ and pulse current ranging from 0.3 to $3 \mathrm{~mA}$, without showing any current dependence. The sample temperature was measured by a Cernox resistor thermometer in close proximity to the samples, and the $R(T)$ curves taken during cooling and heating were compared in order to rule out thermal decoupling effects. The resistivity $\rho$ was estimated bt means of the Van der Pauw technique on unpatterned samples. The resistivity values at room temperature are found to be about $300 \mu \Omega \mathrm{cm}$, according to the resistivities reported in literature. The majority of the samples do not show significant transport anisotropy with respect to the two perpendicular measurement directions in-plane. The $\rho(T)$ curves were linearly interpolated, smoothed by the Savitzky-Golay method and finally derived to obtain the $\mathrm{d} \rho / \mathrm{d} T$. We did not observe any significant variation of the $\mathrm{d} \rho / \mathrm{d} T$ curves by comparing measurements performed in different setups or by changing the order of the interpolation/smoothing/derivation operations.

\section{Results}

The samples grown with different deposition parameters show single orientation, as confirmed by XRD $\theta-2 \theta$ scans, with a large variation of the out-of-plane lattice constant $\left(c_{\mathrm{pc}}=d_{110}=0.393-0.396 \mathrm{~nm}\right)$, ranging from the bulk value to slightly elongated values. Most of the samples show clear size effect oscillations in the $\theta-2 \theta$ scan, meaning that the crystallographic planes are coherent along the film thickness (see for example the intensity oscillation in the RSM around the (001) reflection reported in figures 1(a) and (b), top). Conversely, the samples characterized by bulk lattice parameter lose coherence along their thickness due to structural relaxation, and no oscillations are detected. The in-plane matching of film and substrate is verified by RSM shown in the bottom part of figure 1, showing the (103) reflection of STO and the $(332)_{\circ} /(420)$ 。 reflection of SRO, as we cannot exclude the presence of in-plane twinning. In figure 1 we show the (001) (top) and (103) (bottom) RSM of an optimized sample (figure 1(a)), and of two samples grown at lower oxygen pressure $(0.1 \mathrm{mbar})$, a fully strained one (figure 1(b)) and a partially relaxed one (figure 1(c)), whose structural parameters are reported in table 1 . The 


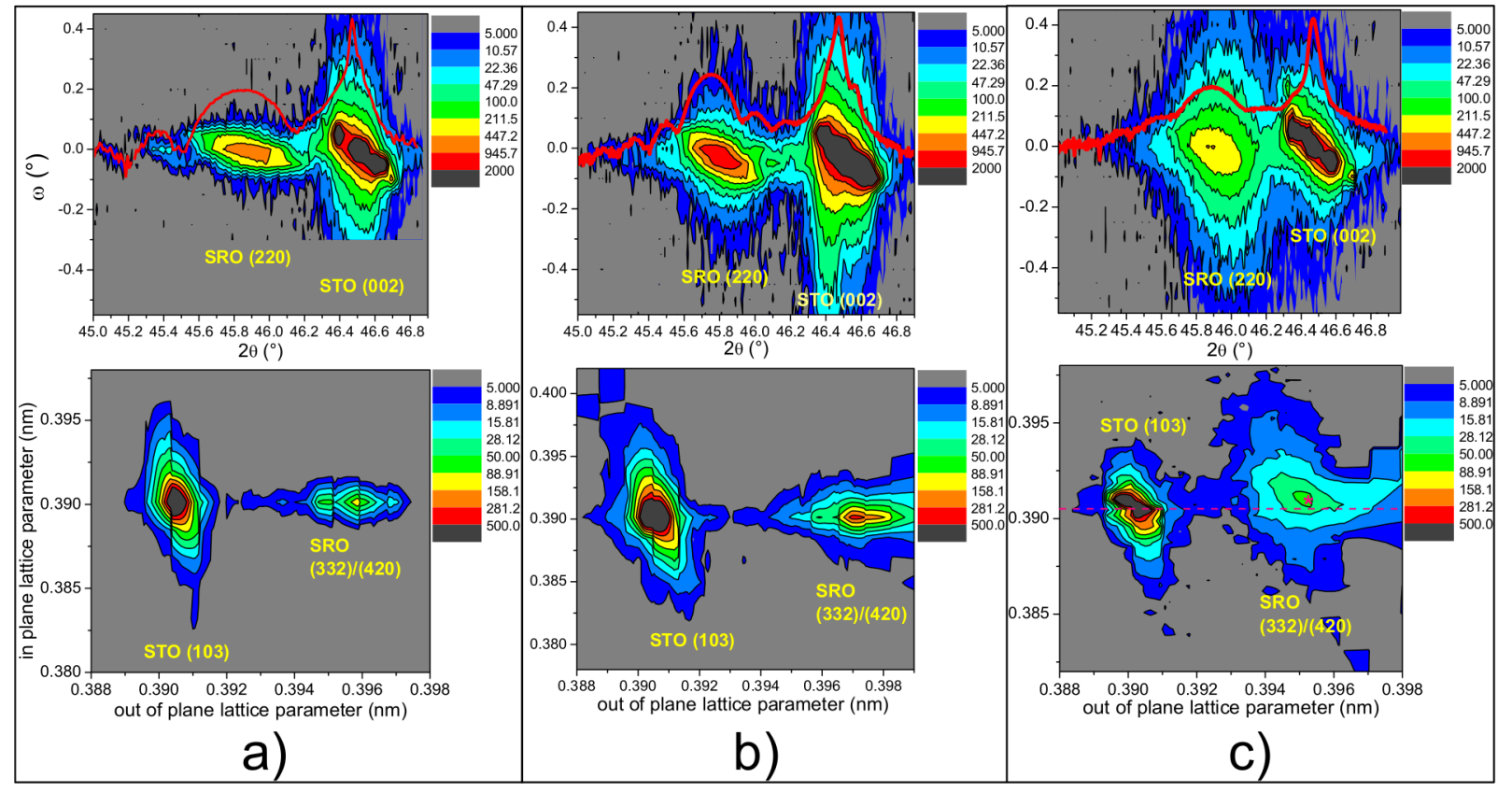

Figure 1. RSM of three selected samples around the (002) (top) and (103) (bottom) substrate reflections. The (103) RSM has been converted to length units. The red line in the top figures is the intensity profile for $\Delta \omega=0$ (arbitrary logarithmic units). (a) An optimized sample; (b) a sample grown at low $\mathrm{O}_{2}$ pressure; (c) a partially relaxed sample: notice that intensity oscillations are not observed, though some remnant features can be observed in the intensity profile that are absent in fully relaxed samples. The dashed line in the bottom figure marks the substrate in-plane lattice parameter, while the asterisk marks the sample's peak, indicating a larger value.

Table 1. Oxygen deposition pressure $P$, laser fluence/frequency $E / f$, deposition rate and structural parameters of the samples of figure 1: out-of-plane pseudocubic lattice constant $c_{\mathrm{pc}}$, in-plane pseudocubic lattice constant $a_{\mathrm{pc}}\left(a_{\text {STO }}\right.$ indicates in-plane matching of film and substrate), rocking curve full width half maximum (FWHM), thickness. All samples have been deposited with same substrate temperature of $720^{\circ} \mathrm{C}$.

\begin{tabular}{llllllll}
\hline Sample & $P(\mathrm{mbar})$ & $\begin{array}{l}E / f \\
(\mathrm{~mJ}, \mathrm{~Hz})\end{array}$ & $\begin{array}{l}\text { Rate } \\
\left(\mathrm{nm} \mathrm{s}^{-1}\right)\end{array}$ & $c_{\mathrm{pc}}(\mathrm{nm})$ & $a_{\mathrm{pc}}(\mathrm{nm})$ & $\begin{array}{l}\text { FWHM } \\
(\mathrm{deg})\end{array}$ & $\begin{array}{l}\text { Thick. } \\
(\mathrm{nm})\end{array}$ \\
\hline $\mathrm{a}$ & 0.2 & $200 / 3$ & 0.04 & $0.3956 \pm 0.0005$ & $a_{\text {STO }}$ & $0.07 \pm 0.01$ & 26.8 \\
$\mathrm{~b}$ & 0.1 & $200 / 3$ & 0.08 & $0.3963 \pm 0.0005$ & $a_{\text {STO }}$ & $0.09 \pm 0.01$ & 55.5 \\
$\mathrm{c}$ & 0.1 & $170 / 2$ & 0.045 & $0.3952 \pm 0.0005$ & $0.391 \pm 0.001$ & $0.11 \pm 0.01$ & 45.0 \\
\hline
\end{tabular}

narrower rocking curve is obtained for the optimized sample, while the relaxed sample is characterized by wide peaks as evident in the $2 \theta-\Delta \omega$ map (figure 1(c)). The samples grown at the lowest $\mathrm{O}_{2}$ pressure $(0.1 \mathrm{mbar})$ are characterized by the largest lattice parameters of the series $\left(c_{\mathrm{pc}}=0.3960-0.3963\right.$ in fully strained samples as the one in figure 1(b)). We believe that the elongation of the $c_{\mathrm{pc}}$ in the samples grown at 0.1 mbar cannot originate from strain only, as in epitaxially matched samples, such an expansion of $c_{\mathrm{pc}}$ would require a Poisson ratio above 0.35 , while in SRO the reported Poisson ratios range between 0.3 and 0.33 , typical values found in oxides [14]. Furthermore we were unable to obtain relaxed samples with bulk lattice parameter at this pressure. We observe that at $0.1 \mathrm{mbar}$ the highest growing rate per pulse is obtained $(0.02-0.03 \mathrm{~nm} /$ pulse versus $0.015 \mathrm{~nm} /$ pulse at higher pressure), and we attribute this occurrence to the weaker braking effect of the background pressure on the plasma plume [15]. Expanded lattice parameter phases have been found in SRO in the form of thin film grown by PLD with different deposition parameters [16, 17], for instance when low deposition pressure allows energetic ion bombardment [18]. However $c_{\mathrm{pc}}$ of the expanded lattice parameter phase reported in $[17,18]$ is much larger $(0.400-0.404 \mathrm{~nm})$ than the ones we observe in our $0.1 \mathrm{mbar}$ films. It has been demonstrated both in thin film by PLD [16] and in bulk samples [19] that, in $\mathrm{SrRu}_{1-x} \mathrm{O}_{3}$, the unit cell volume increases with increasing $\mathrm{Ru}$ vacancies $x$. We then argue that in our samples, the high energy of the species reaching the substrate might favor the re-evaporation of the highly volatile $\mathrm{Ru}$ oxides, yielding $\mathrm{Ru}$-deficient phases with slightly expanded lattice parameters.

By using different deposition conditions, we obtained a wide variety of structural parameters in our samples, thus allowing us to perform a systematic study. In figure 2(a) we report the $\mathrm{d} \rho / \mathrm{d} T$ curves of samples with different characteristics as a function of the reduced temperature $t=$ $\left(T-T_{P}\right) / T_{P}$, where $T_{P}$ is the temperature of the resistivity anomaly, that according to [9] has the form:

$$
\frac{\mathrm{d} \rho}{\mathrm{d} T}=C\left(T-T_{P}\right)^{-\lambda}+c, \quad T>T_{P}
$$



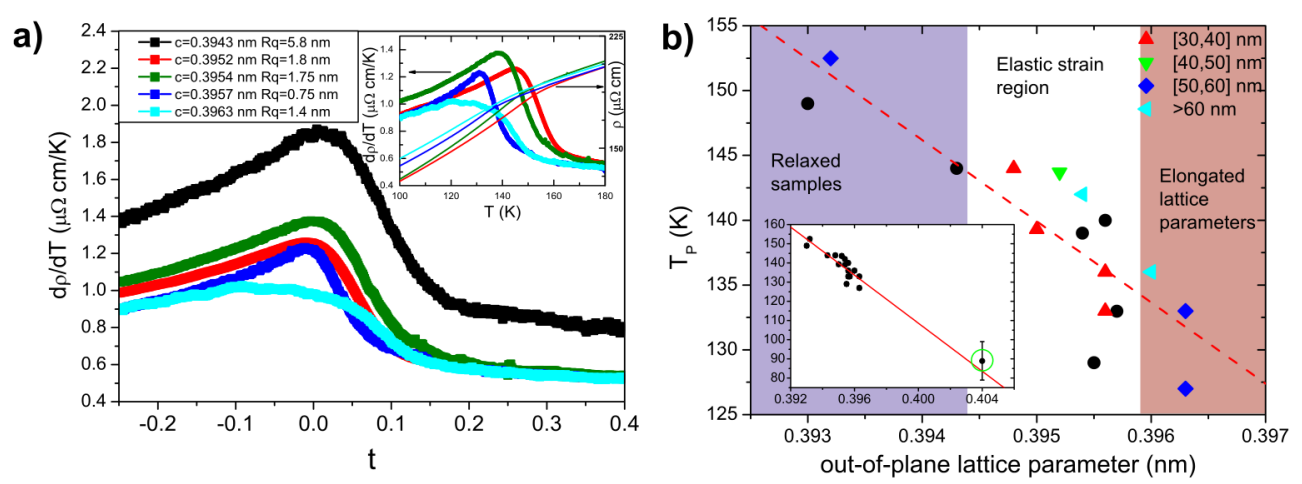

Figure 2. (a) $\mathrm{d} \rho / \mathrm{d} T$ curves plotted as a function of reduced temperature $t$ for different samples, whose out-of-plane lattice parameter (c) and surface roughness $R_{q}$ are reported in the legend. The inset reports some of the $\mathrm{d} \rho(T) / \mathrm{d} T$ curves as a function of temperature (left scale) and the corresponding $\rho(T)$ curves (thin line, right scale). (b) Evolution of the $\mathrm{d} \rho / \mathrm{d} T$ peak temperature $T_{P}$ as a function of the out-of-plane lattice parameter. Different symbols indicate the thickness of the samples, showing that the relationship holds independently from thickness. The red dashed line is linear fit to guide the eyes. In the inset the same data and the same line is reported together with the $T_{P}$ value obtained for a sample of [18] (green circle).

$$
\frac{\mathrm{d} \rho}{\mathrm{d} T}=C^{\prime} \ln \left|T-T_{P}\right|+c^{\prime}, \quad T<T_{P} ;
$$

where $\lambda$ is the critical exponent, $C, C^{\prime}$ are proportionality factors, and $c, c^{\prime}$ are the constant background values of $\mathrm{d} \rho / \mathrm{d} T$ far from the transition. In most cases $T_{P}$ coincides with the temperature of the maximum of the $\mathrm{d} \rho / \mathrm{d} T$ curve, as we have verified by fitting the right (left) part of the curves with respect to the maximum with equations (1) and (2), using $C, T_{P}, c, \lambda\left(C^{\prime}, T_{P}, c^{\prime}\right)$ as fitting parameters. According to the theory of critical resistivity fluctuations in magnetic metals [10], the resistivity anomaly occurs at the Curie temperature, then $T_{P}$ ideally coincides with the $T_{\mathrm{C}}$ value. However, in nanometric thin films, a discrepancy between the onset temperature of magnetization and the peak in the $\mathrm{d} \rho / \mathrm{d} T$ curve has been observed. This can be attributed to the broadening of the magnetic transition due to the coexistence of different phases $[17,19]$.

From figure 2(a) we can observe that the relaxed sample $\left(c_{\mathrm{pc}}=0.3943 \pm 0.0005 \mathrm{~nm}\right)$ is characterized by higher values of $\mathrm{d} \rho / \mathrm{d} T$ far from the transition if compared to the strained samples, corresponding to a higher resistivity $(\approx 400 \mu \Omega \mathrm{cm}$ at room temperature), as a consequence of its granular microstructure (as shown in the following). Also, the sample with elongated lattice parameter $\left(c_{\mathrm{pc}}=0.3963 \pm 0.0005 \mathrm{~nm}\right)$ is characterized by a wider peak if compared to the other samples, which may indicate a degradation of the magnetic properties related to the anomalously large lattice parameter. In figure 2(b) we report the $T_{P}$ values as a function of $c_{\mathrm{pc}}$ for samples deposited in various deposition conditions. The $T_{P}$ shows a linear correlation with the lattice parameter in strained samples as well as in relaxed and elongated lattice parameter ones. To verify that this correlation holds also for the expanded structure reported in $[17,18]$, we extracted the $\rho(T)$ data from [18], for a $c_{\mathrm{pc}}=0.404 \mathrm{~nm}$ sample, and we obtained the corresponding $T_{P}$ value. In the inset of figure 2(b) we replot the data with the new data point (circled), showing that it falls on the same line as our data.

We varied the deposition conditions in order to minimize the surface roughness of the films. As expected, the roughness of relaxed samples $\left(R_{q}>5 \mathrm{~nm}\right)$, characterized by bulk-like out-of-plane lattice parameter, is much higher than the one of strained samples (we found $R_{q} \leq 2 \mathrm{~nm}$ for sample thickness up to $130 \mathrm{~nm}$ ), but no further correlation between roughness and lattice parameter is found. For selected samples the AFM topography is reported, namely a relaxed sample (figure 3(a)) and two strained samples obtained with different deposition parameters (figures 3(b) and (c)). The morphology of the relaxed sample reveals a granular structure with grains characterized by facets along the $\langle 110\rangle$ STO directions. Conversely the strained samples are characterized by $3 \mathrm{D}$ islands, more or less disordered, depending on deposition conditions. The lowest value of $R_{q}$ we have obtained is of the order of 2 out-of-plane lattice parameters (see for example the sample of figure 3(c)), while the expected value in samples characterized by $2 \mathrm{D}$ steps (indicating ordered layer by layer or step flow growth) [2] is $R_{q} \approx 1$ lattice parameter. In figure 3(c) the islands are organized along one direction, originating a terrace structure often observed on SRO samples after the earliest stages of growth [20]. It has been shown that in particular growth conditions [21], 3D islands preferentially nucleate along the substrate miscut-induced steps (for the sample of figure 3(c) the miscut angle of the substrate determined by $\mathrm{XRD}$ is $\approx 0.12^{\circ}$ ), generating a finger structure that eventually merges and converts the growth to $2 \mathrm{D}$ steps above a critical thickness (about $40 \mathrm{~nm}$ in case of [20], that is larger than the thickness of the samples shown in figure 3).

From figure 2(a), it can be observed that the sharpness of the $\mathrm{d} \rho / \mathrm{d} T$ maximum varies from sample to sample, and we then want to investigate the relationship between this feature and the structure of the samples. In order to study the critical behavior of the resistivity, we have performed a non-linear fit (by Levenberg-Marquardt fitting routine) of the $\mathrm{d} \rho / \mathrm{d} T$ curves for $t>0$, by reformulating equation (1) as follows:

$$
\frac{\mathrm{d} \rho}{\mathrm{d} T}=K t^{-\lambda}+c
$$

with $K$ and $\lambda$ as free fitting parameters, while the constant non-magnetic background $c$ value was fixed accordingly to 


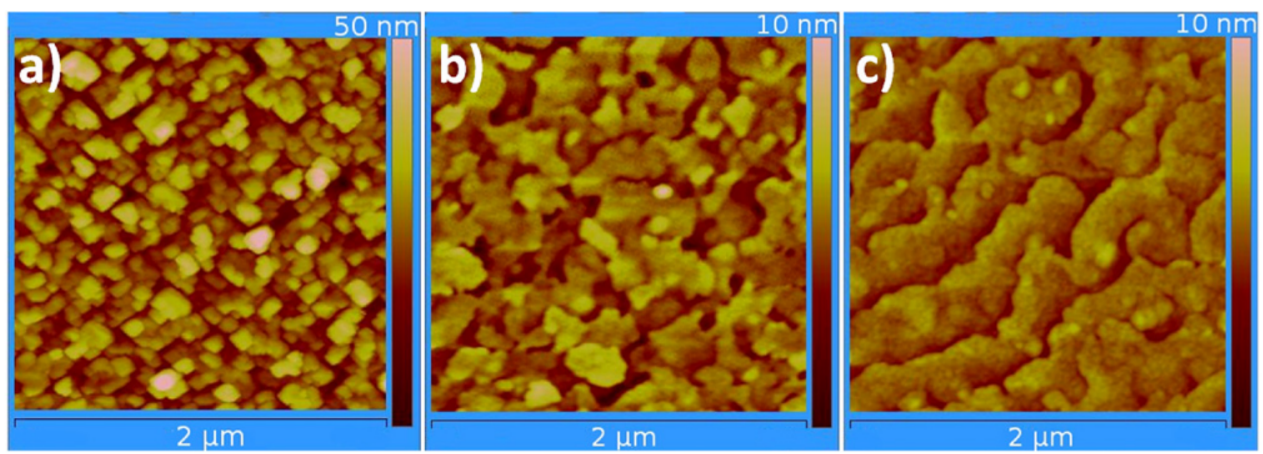

Figure 3. Tapping mode AFM topography of three samples: (a) relaxed sample $\left(R_{q}=7.2 \mathrm{~nm}\right)$, (b) and (c) strained samples $\left(R_{q}=1.2\right.$, $0.75 \mathrm{~nm}$ respectively). Notice the different color scales. The thickness of the samples is: (a) $23 \mathrm{~nm}$, (b) $28 \mathrm{~nm}$ and (c) $27 \mathrm{~nm}$.

the $\mathrm{d} \rho / \mathrm{d} T$ value for $t \gg 0$. By using equation (3), as a function of the reduced temperature, we limit the number of fitting parameters (see equation (1)), allowing a more reliable determination of the critical exponent $\lambda$, as dependences between the parameters occur when using a large number of fitting parameters. Furthermore, with this approach we can choose equivalent fitting intervals for all the samples, depending on the relevant parameter in phase transitions, i.e. the reduced temperature $t$ [22]. The fitting interval ranges from $t=0.01-0.05$ to $0.25-0.30$, depending on the sample in order to avoid the rounded part of the curve for $t \rightarrow 0$. As a consequence of the pronounced rounding of the curves, we excluded the elongated lattice parameter samples from this analysis. The obtained $\lambda$ values are plotted versus roughness in figure 4 . For strained samples (in the elastic strain region of figure 2(b)) the critical exponent is $0.5<\lambda<1.1$, while for relaxed samples, $\lambda$ saturates at $1 \pm 0.1$ (see the inset of figure 4), in agreement with the values reported by Klein et al [9]. Restricting to the strained samples, we find that the critical exponent value shows correlation to the sample roughness, approaching the 'mean-field' value $\lambda=1 / 2$ as the roughness decreases. On the other hand no correlation is found with other structural parameters such as lattice parameter or thickness.

\section{Discussion}

The $T_{P}$ suppression as a function of the lattice parameter is in agreement with other reports on thin films showing that as the strain is released (by substrate selective etching [4] or by increasing the film thickness [23]), the $\rho(T)$ anomaly shows at higher temperature. It is indeed found that the $T_{\mathrm{C}}$, the magnetic moment and the sharpness of the magnetic transition are enhanced when the strain is relieved [4]. As discussed in section 1 , the $\mathrm{RuO}_{6}$ octahedron rotations affect the hybridization of $\mathrm{Ru}$ and $\mathrm{O}$ orbitals, thus modifying the density of states at the Fermi level, and double the unit cell, leading to opening of pseudo-gaps. This affects the magnetism, and consequently the transport properties of SRO. Linearized augmented plane wave calculations [3] show that the magnetic moment in SRO is suppressed if a cubic structure is used in calculation $\left(\mathrm{Ru}-\mathrm{O}-\mathrm{Ru}\right.$ is $\left.180^{\circ}\right)$, reaches its maximum for the actual SRO octahedron rotations, and then decreases again for increasing rotation to disappear in the case

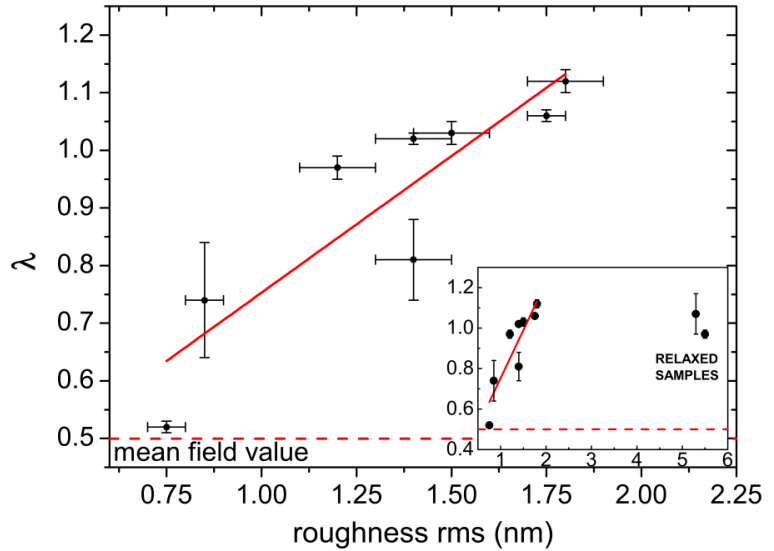

Figure 4. Plot of the $\lambda$ values, obtained by the fitting of the $\rho(T)$ curves, versus the sample roughness. The error associated with $\lambda$ is three times the standard deviation returned by the fitting routine, or half of the dispersion of the $\lambda$ values obtained from different measurements (points with large error bars, in presence of anisotropic $\rho(T)$ ). The full line is a linear fit to the data, to guide the eyes, while the dashed lines indicates $\lambda=1 / 2$. In the inset the data are reported together with the values obtained for the relaxed samples.

of the $\mathrm{CaRuO}_{3}$ structure. In Ru-deficient bulk samples, the main effect of vacancies on the structure is the unfolding of the $\mathrm{Ru}-\mathrm{O}-\mathrm{Ru}$ bonds (the angle of the bond comes closer to $180^{\circ}$ ), so that the lattice parameter expands, and at the same time a suppression of the $T_{\mathrm{C}}$ is observed [19].

Our data show that the $T_{P}$ is linearly correlated to the out-of-plane lattice parameter, regardless if we are looking at a pure elastic strain induced distortion of the elongation of lattice parameters induced by vacancies (in 0.1 mbar samples).

The theory of resistivity critical fluctuations that was first introduced by De Gennes and Friedel [24], and subsequently by Fisher and Langer [10], has been then extended to cope with different situations (metals, semiconductors, antiferromagnets) [25] and with the breakdown of Boltzmann approach (expected to occur in a bad metal such as SRO) [26]. Within the Boltzmann approach, and as long as the Fermi wavevector is much larger than the inverse correlation length, the proportionality between $\mathrm{d} \rho / \mathrm{d} T$ and $C_{p}$ still holds above the Ginzburg temperature $t_{\mathrm{G}}$, but with the classical mean-field exponent $\alpha^{\prime}=1 / 2$, as Gaussian fluctuations dominate instead 
of the critical ones [25]. Measurements performed on high-quality SRO single crystals [8], seem to confirm this picture, as both the specific heat and magnetization anomaly could be fully described by means of mean-field behavior, thus indicating that true critical behavior is attained in SRO only at very low reduced $t(t<0.0003)$. The proportionality between $\mathrm{d} \rho / \mathrm{d} T$ and $C_{p}$ was also verified, thus yielding $\lambda=$ $\alpha^{\prime}=1 / 2$. The discrepancy between the results obtained on thin films (Ising critical exponents for the magnetization, and $\lambda=0.9$ ) [9] are attributed to sample inhomogeneities in thin film samples (smearing of the transition). Due to the bad metal nature of SRO, it may be objected that the Boltzmann approach cannot be applied in this case. However the same (i.e. mean field) critical exponent $(\lambda=1 / 2)$ is obtained from approaches that go beyond Boltzmann equation formalism [26]. We then expect that in ordered samples the $\mathrm{d} \rho / \mathrm{d} T$ critical exponent approaches the value $1 / 2$, while in disordered samples, where dislocation, vacancies, grain boundaries or multi-phases are present, we expect to observe a larger $\lambda$, according to the literature. This is indeed what we find in our samples as a function of roughness (see figure 4). When an ordered growth is ensured by favorable conditions, ordered single-phase samples with low roughness are obtained, and the corresponding $\lambda \rightarrow 1 / 2$. Conversely, in samples characterized by a disordered microstructure, such as the ones displaying islands superimposed on multiple levels and deep pits (see figure 3(b)), or even a granular structure (figure 3(a)), we observe larger $\lambda$ values.

\section{Conclusions}

In our study we show that the critical behavior of the $\rho(T)$ in SRO thin films reveals direct correlation between transport properties and structural features, such as the lattice parameters and the surface roughness. Our data demonstrate that the $T_{P}$ is a good measure of the average lattice distortion, being dependent on the lattice parameter only, regardless of the vacancy content and of the more or less disordered microstructure. In turn this indicates that lattice effects, most probably through the modification of the $\mathrm{Ru}-\mathrm{O}-\mathrm{Ru}$ bond angle, dominate over stoichiometry and disorder effects in determining the suppression of the temperature of the resistivity anomaly, and correspondingly, of the $T_{\mathrm{C}}$.

At the same time the value of the critical exponent $\lambda$ is a measure of the microscopic order of the samples. Its value is correlated to the sample roughness for the strained samples, and saturates to $0.9-1$ for relaxed granular samples. Our result suggests that the $\lambda=1 / 2$ value is expected in ordered SRO and then explains the discrepancy between the critical exponents found in single crystals and thin films [8] in terms of inhomogeneity of the samples.

Our results demonstrate once again the correlation between structure and transport properties in SRO thin films, with a particular focus on the critical properties as a function of structure and disorder, and offer a simple way to test SRO sample quality via electrical transport measurements.

\section{Acknowledgments}

AG wishes to thank R Ciancio for experimental support in the sample characterization, and acknowledges partial financial support through exchange grants from the Centre National de la Recherche Scientifique through a PICS program (Projets internationaux de coopération scientifique).

\section{References}

[1] Koster G, Klein L, Siemons W, Rijnders G, Dodge J S, Eom C B, Blank D H A and Beasley M R 2012 Rev. Mod. Phys. 84253

[2] Estève D, Maroutian T, Pillard V and Lecœur Ph 2011 Phys. Rev. B 83193401

[3] Mazin I I and Singh D J 1997 Phys. Rev. B 562556

[4] Gan Q, Rao R A, Eom C B, Garrett J L and Lee M 1998 Appl. Phys. Lett. 72978

[5] Orgiani P, Aruta C, Balestrino G, Lavanga S, Medaglia P G and Tebano A 2002 Eur. Phys. J. B 2623

[6] Allen P B, Berger H, Chauvet O, Forro L, Jarlborg T, Junod A, Revaz B and Santi G 1996 Phys. Rev. B 534393

[7] Gurvitch M 1981 Phys. Rev. B 247404 and references therein

[8] Kim D, Zink B L, Hellman F, McCall S, Cao G and Crow J E 2003 Phys. Rev. B 67100406

[9] Klein L, Dodge J S, Ahn C H, Reiner J W, Mieville L, Geballe T H, Beasley M R and Kapitulnik A 1996 J. Phys.: Condens. Matter 810111

[10] Fisher M E and Langer J S 1968 Phys. Rev. Lett. 20665

[11] Gan Q, Rao R A and Eom C B 1997 Appl. Phys. Lett. 701962

[12] Herranz G, Sánchez F, Fontcuberta J, García-Cuenca M V, Ferrater C, Varela M, Angelova T, Cros A and Cantarero A 2005 Phys. Rev. B 71174411

[13] Orgiani P, Petrov A Yu, Adamo C, Aruta C, Barone C, De Luca G M, Galdi A, Polichetti M, Zola D and Maritato L 2006 Phys. Rev. B 74134419

[14] Vasta G, Jackson T J, Frommhold A, Bowen J and Tarte E J 2011 J. Electroceram. 27176

[15] Sambri A, Amoruso S, Wang X, Miletto Granozio F and Bruzzese R 2008 J. Appl. Phys. 104053304

[16] Yoo Y Z, Chmaissem O, Kolesnik S, Dabrowski B, Maxwell M, Kimball C W, McAnelly L, Haji-Sheikh M and Genis A P 2005 J. Appl. Phys. 97103525

[17] Rundqvist P, Vorobiev A, Gevorgian S, Khamchane K and Ivanov Z 2003 J. Appl. Phys. 931291

[18] Maria J-P, Trolier-Mc Kinstry S, Schlom D G, Hawley M E and Brown G W 1998 J. Appl. Phys. 841374

[19] Dabrowski B, Chmaissem O, Klamut P W, Kolesnik S, Maxwell M, Mais J, Ito Y, Armstrong B D, Jorgensen J D and Short S 2004 Phys. Rev. B 70014423

[20] Sánchez F, Herranz G, Infante I C, Ferrater C, García-Cuenca M V, Varela M and Fontcuberta J 2006 Prog. Solid State Chem. 34213

[21] Sánchez F, Lüders U, Herranz G, Infante I C, Fontcuberta J, García-Cuenca M V, Ferrater C and Varela M 2005 Nanotechnology 16 S190

[22] Kirtley J R, Tsuei C C and Tafuri F 2003 Phys. Rev. Lett. 90257001

[23] Herranz G, Martínez B, Fontcuberta J, Sánchez F, Ferrater C, García-Cuenca M V and Varela M 2003 Phys. Rev. B 67174423

[24] De Gennes P G and Friedel J 1958 J. Phys. Chem. Solids 471

[25] Alexander S, Helman J S and Balberg I 1976 Phys. Rev. B 13304

[26] Timm C, Raikh M E and Oppen F 2005 Phys. Rev. Lett. 94036602 